\title{
Systems Research and Development at VMware
}

\author{
Stephen Alan Herrod \\ Chief Technology Officer, VMware \\ sherrod@vmware.com
}

\begin{abstract}
Over the last twelve years, VMware has grown from a small startup with an interesting idea to an $8000+$ person company that is changing the landscape of the datacenter and how IT happens in the enterprise. The VMware story has been one of intense technological innovation delivered consistently as a series of products and releases. We will briefly look at the history of innovation at VMware and how we have balanced research, advanced development and product delivery to set the context for the papers in this issue.
\end{abstract}

\section{INTRODUCTION}

Cloud Computing is one of the most discussed areas in computer system software today, seen as the next big wave of approaches to computing. Virtualization is proving to be the key enabler of cloud computing, putting VMware in a unique position to help define and deliver the technologies that will enable its pervasive use. There are many forms of virtualization, but VMware has largely focused on hardware virtualization. By creating a level of indirection between industry-standard hardware and operating systems, virtualization has led to innovation in hardware resource optimization, security, and application availability. It has also enabled important new datacenter capabilities such as softwarebased fault-tolerance and the live migration of applications between physical machines or even geographies.

In this special issue of Operating System Review, our engineering team shares our history of researching and developing these new technologies and how they have already played a role in disrupting the traditional approach to computing. First, let's provide a historic backdrop of VMware and the culture that led to its creation.

Dr. Mendel Rosenblum, associate professor of Computer Science at Stanford University (and my Ph.D. advisor), encouraged his research group to focus on machine virtualization as a technique for utilizing servers with enormous computing power. Virtualization was an incredibly popular approach to mainframe time-sharing, largely driven by IBM throughout the 60's and 70's. The interesting research questions were "Why has it become less pervasive in the 80's and 90's?" and "Could we possibly implement virtualization in the modern world of industry-standard PCs, servers, and operating systems?" With this backdrop, Dr. Rosenblum and his students launched several related projects into this space. (One such project was named "Disco"... like virtualization, a cool idea from the 70's that seemed ready for a comeback!). From these academic beginnings, Dr. Rosenblum, two of his students, a colleague, and industry-veteran Diane Greene created VMware with the goal of converting this unproven research into products.
After moving to rather austere facilities in nearby Palo Alto, VMware's initial focus was determining whether it was even possible to virtualize the $\mathrm{x} 86$ architecture. Unlike prior machine virtualization successes, the $\mathrm{x} 86 \mathrm{CPU}$ was accepted to have an "unvirtualizable" architecture, lacking the processor features traditionally required for virtualization. Coupled with a complex instruction set, instruction execution full of side effects, and sparse documentation, this was a significant development barrier. The paper entitled "The Evolution of an x86 Virtual Machine Monitor" takes you on a journey through this original research and how the team worked tirelessly to create the first functional and performant virtual machine monitor for the $\mathrm{x} 86$ architecture and how they were able to continually simplify and speed up the design as the CPU manufacturers added explicit hardware changes to make this job easier.

VMware's early success stemmed from allowing access to applications that would not otherwise be available to users (e.g. Windows on Linux). Early adopters of the technology used it for a variety of needs: to run Microsoft Windows applications on their Linux desktops, to more easily test application compatibility across a variety of Microsoft Windows versions and service packs, and to try out new operating systems (anyone remember Plan9, BeOS?) without polluting their computer's hard drive.

While VMware's commercial success grew in the PC space, VMware's researchers had shifted their focus onto the next daunting research challenge. Could the core technology be robust and performant enough to virtualize higher-end servers and run across all of the servers, storage, and networking devices that make up modern datacenters? These challenges have been VMware's focus for more than a decade now, and this special issue includes papers covering just a small fraction of the interesting research work we've been involved with, as we've brought datacenter virtualization to more than 230,000 customers around the world.

While our earliest work examined how to run a single virtual machine (VM) well, the datacenter opportunities require running a large number of VMs on a smaller number of physical machines. Resource management has been a top focus for VMware since the first release of our server products. In this issue we're including more advanced research in this space with "Online Cache Modeling for Commodity Multicore Processors". In addition to running these VMs with fair resource allocation, we've also found great ways to protect the VMs, giving them higher levels of reliability when virtualized than when running directly on hardware. "The Design of a Practical System for Fault-Tolerant Virtual Machines" describes one such approach to this increased reliability.

As VMware products have moved even deeper into the datacenter, we've extended our research and development off the 
motherboard and squarely into the realm of high-speed networking. In "Virtual Networking", our engineers share experiences extending virtualization into the realm of high-speed $\mathrm{I} / \mathrm{O}$, and in "Virtualizing Networking and Security in the Cloud" they show how we can build upon virtualized networking with innovative new approaches to application security. Storage is also essential to the virtualized datacenter, and we've provided a taste of the storage challenges we're working on in "Virtual Machine File System" and "Scalable Virtual Machine Storage using Local Disks". All of these new capabilities provide real value to customers, and we've always focused on how to do so while minimizing performance overheads. "Virtualization Performance - Perspectives and Challenges Ahead" addresses this topic, covering many unique challenges of this environment.

The above research has focused on the core platform for running virtual machines, but the commercial success of virtualization is really due to making management of these massive datacenter environments substantially easier. Systems management for this new world is a major focus of research and development at VMware, and you can get a glimpse into the ways we approach these large environments in "Challenges in Building Scalable Virtualized Datacenter Management". We have also ramped up our efforts to look at how virtualization can enable a competitive marketplace with multiple choices as to where applications can be hosted. Such an ecosystem for both new and existing applications is a key enabler of truly pervasive cloud computing, and is the subject of "Enabling a Marketplace of Clouds: VMware's vCloud Director"
And as with all research efforts into broad new spaces, our investigations into virtualization of PCs and datacenters have led to even more open questions in adjacent areas, and we include several such examples in this issue. In "vApp: A Standards-based Container for Cloud Providers", we investigate new opportunities for application packaging in a fully virtualized world. "Virtualizing High Performance Computing" explores how applicable these technologies are to the realm of scientific- and grid-computing. And in "The VMware Mobile Virtualization Platform: is that a hypervisor in your pocket?" we share our early efforts to apply many of these same technologies to the wild world of smart phones.

I hope you get a taste of the interesting research problems that VMware has faced over the past 13 years. What I'm most proud about is that the engineering team has taken on these challenges with a focus on rapidly delivering the products that solve them. This ability to convert core research to enterprise-quality products is unique, and has proven key to virtualization's success. Putting it in perspective, today more than half of all $\mathrm{x} 86$ server applications are virtualized, an amazing achievement considering the early days of determining whether virtualization of the x86 was possible at all.

I'd like to close this introduction with a sincere thank you to ACM SIGOPS for giving us the opportunity to participate in this special issue. I hope you enjoy the papers, and here's to the even more exciting challenges that surely lie ahead. 